\title{
Predictive Factors for the Long-Term Deterioration of Pulmonary Function in Interstitial Lung Disease Associated with Anti-Aminoacyl-tRNA Synthetase Antibodies
}

\author{
Hideaki Yamakawa ${ }^{a}$ d Eri Hagiwara ${ }^{a}$ Hideya Kitamuraa, d Tae Iwasawab \\ Ryota Otoshi $^{a}$ Naoto Aiko ${ }^{a}$ Takuma Katano $^{a}$ Ryota Shintani ${ }^{a}$ Satoshi lkeda ${ }^{a}$

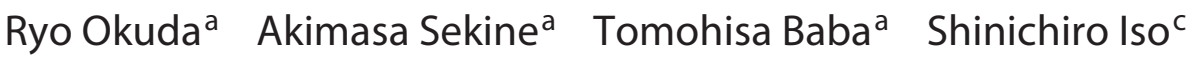 \\ Kazuyoshi Kuwano ${ }^{d}$ Shinji Sato $^{\text {e Takashi Oguraa }}{ }^{a}$

\begin{abstract}
a Department of Respiratory Medicine, Kanagawa Cardiovascular and Respiratory Center, Yokohama, Japan;
${ }^{b}$ Department of Radiology, Kanagawa Cardiovascular and Respiratory Center, Yokohama, Japan; ' Department of Radiology, Yokohama Rosai Hospital for Labor Welfare Corporation, Yokohama, Japan; d Department of Respiratory Medicine, Tokyo Jikei University Hospital, Tokyo, Japan; 'Department of Rheumatology, Tokai University Hospital,
\end{abstract} \\ Isehara, Japan
}

For editorial comment see p. 207

\section{Keywords}

Antisynthetase syndrome $\cdot$ Interstitial lung disease .

Long-term disease behavior · Middle lobe traction

bronchiectasis

\begin{abstract}
Background: Little has been reported on long-term pulmonary function trends among patients with interstitial lung disease associated with anti-aminoacyl-tRNA synthetase antibodies (ARS-ILD). Objectives: To clarify the factors predictive of progression in ARS-ILD based on patients' initial clinical and radiological features. Methods: The clinical courses of 88 patients with $>1$ year of follow-up data on pulmonary function tests (PFTs) were retrospectively analyzed. Disease behavior was categorized into three groups: (1) improved or (2) worsened (defined as increases or decreases, respectively, of $>10 \%$ in forced vital capacity and $>15 \%$ in
\end{abstract}

\%diffusing capacity of lung carbon monoxide) or (3) stable based on PFT changes compared between 1-year results as the initial data and results at 3 years to assess the long-term course. Results: In the initial course of 75 patients with ARSILD who received anti-inflammatory therapy within 6 months after diagnosis, 48 patients (64.0\%) improved and 6 patients $(8.0 \%)$ worsened. The radiological patterns in the patients with ARS-ILD included nonspecific interstitial pneumonia (NSIP) in $46.7 \%$ and NSIP with organizing pneumonia overlap in $52.0 \%$ of the cases. One-third of the initially improved patients who worsened over the long-term course were assigned to the unstable group. By multivariate logistic analysis, middle lobe traction bronchiectasis was a significant predictive factor for the patients in the unstable

All work was performed at the Kanagawa Cardiovascular and Respiratory Center.

\section{KARGER}

(C) 2018 S. Karger AG, Base

E-Mail karger@karger.com

www.karger.com/res
Hideaki Yamakawa, MD, $\mathrm{PhD}$

Department of Respiratory Medicine

Kanagawa Cardiovascular and Respiratory Center

6-16-1 Tomioka-higashi, Kanazawa-ku, Yokohama 236-0051 (Japan)

E-Mail hide1144@jikei.ac.jp 
group. Conclusions: Most patients with ARS-ILD receiving anti-inflammatory therapy had improved or remained stable in the first year. However, over the long-term course, some patients worsened despite their initial improvement. Even though the extent of the disease is limited, middle lobe traction bronchiectasis in ARS-ILD may be a useful predictor of poor long-term disease behavior. @ @ 2018 S. Karger AG, Basel

\section{Introduction}

Anti-aminoacyl-tRNA synthetase (anti-ARS) antibodies are known to be specific to polymyositis (PM)/ dermatomyositis (DM) [1-3]. Among patients with antiARS antibodies there is a high frequency of interstitial lung disease (ILD) (ARS-ILD) (range: 71-100\%) [2]. Previous reports have indicated that the clinical characteristics are similar in PM/DM-associated ILD and in ARSILD without PM/DM $[4,5]$. Although some patients with rapidly progressive ARS-ILD have a poor prognosis in the short run, their overall prognosis is relatively good $[6,7]$. However, because of frequent recurrence and ARS-ILD refractory to therapy, some patients progress to pulmonary fibrosis over the long run despite initial improvement [8-10]. Some patients are forced to use home oxygen therapy, while a few undergo lung transplantation in clinical practice.

It is not known which subgroup of patients with ARSILD will suffer long-term deterioration. Therefore, this study aimed to clarify the factors predictive of long-term progression of deteriorating pulmonary function in ARSILD from the viewpoint of the patients' initial clinical and radiological features.

\section{Subjects and Methods}

Study Sample

Our study included 129 consecutive patients diagnosed with ARS-ILD between January 1996 and March 2017 at the Kanagawa Cardiovascular and Respiratory Center. Of those, we excluded 41 patients: 29 who were diagnosed after initiation of medication at another hospital, 11 lacking more than 1 year of follow-up data, and 1 who died within the first year. Thus, 88 patients with clinical, physiological, and radiological data collected for more than 1 year were examined.

The diagnosis of PM/DM was made by rheumatologists according to the criteria of Bohan and Peter [11]. Patients with definite, probable, or possible $\mathrm{PM} / \mathrm{DM}$ were included in the study. Patients diagnosed as having interstitial pneumonia with autoimmune features (IPAF) fulfilled the criteria of the ERS/ATS task force [12]. ILD onset was classified into two types, (1) an acute/ subacute form (rapidly progressive ILD showing deterioration within 3 months) and (2) a chronic form (slowly progressive presentation with gradual deterioration over a period of $>3$ months), according to previous reports $[13,14]$.

\section{Data Collection}

Baseline clinical measurements were obtained within 3 months of the initial diagnosis of ILD at our hospital. A broad panel of autoantibodies was evaluated as part of the initial workup or during the follow-up period. For ARS antibodies (anti-Jo-1, EJ, PL-7, PL$12, \mathrm{OJ}$, and $\mathrm{KS}$ antibodies), routine conserved serum of each patient was analyzed by RNA immunoprecipitation and protein immunoprecipitation assays at the Tokai University School of Medicine, Isehara, Japan.

\section{Radiological Analysis}

Two experienced thoracic radiologists (T. Iwasawa and S. Iso) reviewed all high-resolution computed tomography (HRCT) scans for consensus about the diagnosis of ILD in our hospital without knowledge of the patients' clinical data. The HRCT patterns were classified into nonspecific interstitial pneumonia (NSIP), organizing pneumonia (OP), usual interstitial pneumonia, and NSIP with OP overlap according to previous reports $[6,15]$. NSIP with OP overlap was identified when consolidations were superimposed on a background of ground-glass opacity (GGO) with or without reticulations or traction bronchiectasis [6]. The HRCT findings were interpreted according to the recommendations of the Fleischner Society [16]. Disagreements between the two radiologists after the initial assessment were resolved by discussion.

\section{Pulmonary Function Tests}

Changes in results of pulmonary function tests (PFTs) are presented as the percentage change between the initial value and that measured at 1 year (including at 1 year \pm 6 months). Patients were assigned to the "improved" or "worsened" group based on increases or decreases, respectively, of $>10 \%$ in forced vital capacity (FVC) and $>15 \%$ in \%diffusing capacity of the lung for carbon monoxide (\%DLCO) [17]. Patients who did not meet the criteria for the improved or worsened group were assigned to the "stable" group. In addition, changes in the PFT results of the patients who received anti-inflammatory therapy (i.e., corticosteroid, cyclosporine, and tacrolimus) within 6 months after ILD diagnosis were also analyzed at 3 years, and the results were compared with those at 1 year. Changes in FVC and \%DLCO at 3 years (including at 3 years \pm 6 months) in the patients of the improved group at 1 year were compared with the 1-year PFT results to assess long-term disease behavior. These patients were subsequently categorized into two subgroups: a "stabilization" group, which included stable patients, and an "unstable" group, which included patients who worsened after having initially shown improvement at 1 year.

\section{Statistical Methods}

Categorical baseline characteristics are summarized by frequency and percentage, and continuous characteristics are reported as the mean \pm SD or median (IQR). To detect differences between groups, Fisher's exact test, one-way ANOVA, or the MannWhitney $U$ test was used as appropriate. Regarding the HRCT findings, the $\kappa$ value was calculated for agreement between the two thoracic radiologists for the baseline read. Next, we performed a 


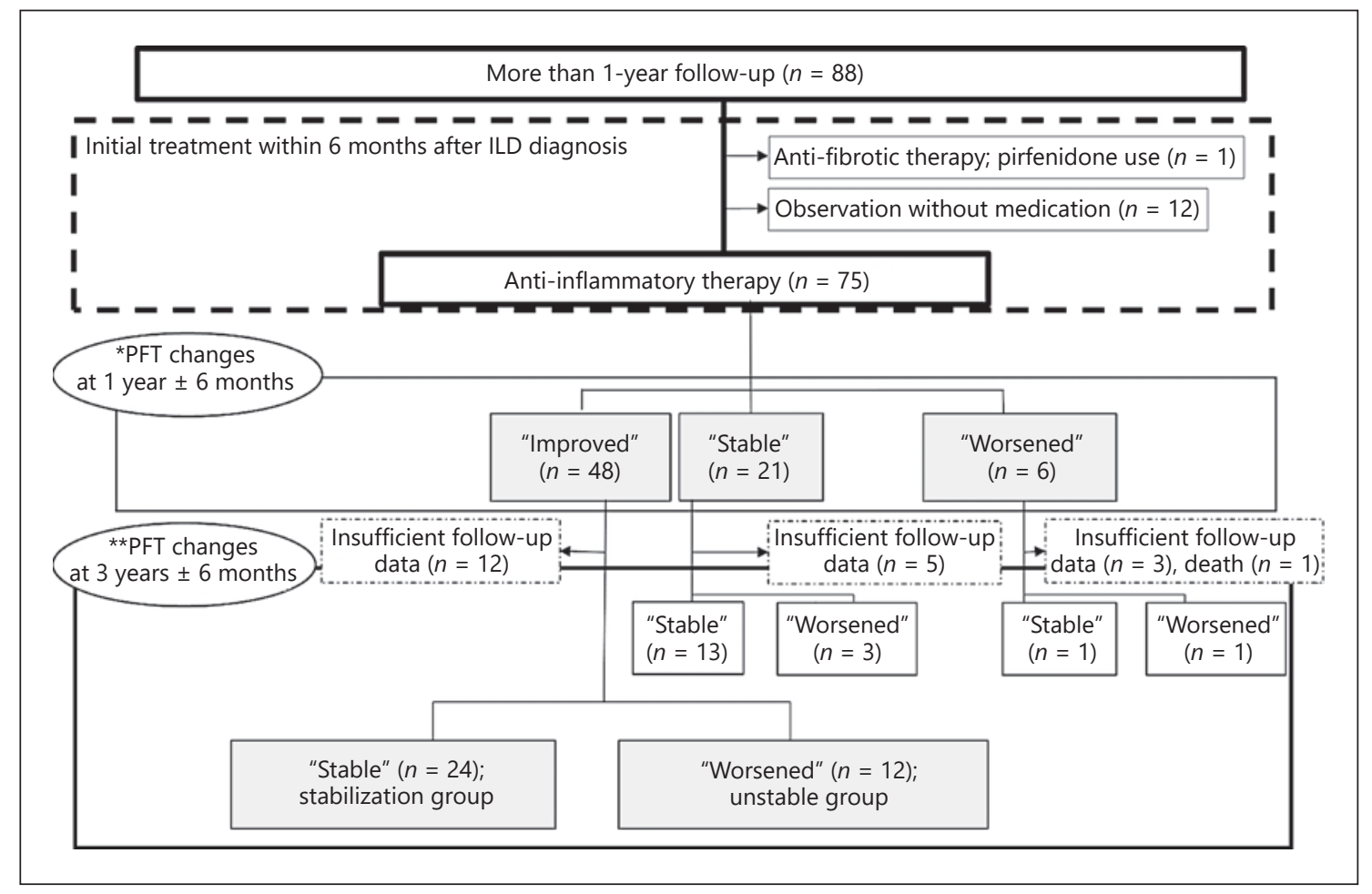

Fig. 1. Clinical courses of the patients with positive anti-aminoacyl-tRNA synthetase antibodies and ILD. ILD, interstitial lung disease; PFT, pulmonary function test.

logistic regression analysis to identify the predictive factors associated with the improved and worsened groups at 1 year, which was considered as the "initial course." Thereafter, the variables that achieved a modest level of statistical significance $(p<0.1$ on univariate analysis) based on the forced entry method were assessed in multivariate analysis. We also performed logistic regression analysis using the same methods to identify the predictive factors associated with the unstable group at 3 years to assess long-term deterioration. We considered $p<0.05$ to represent statistical significance in all analyses. All data were analyzed with SPSS version 22.0 (IBM Japan, Tokyo, Japan).

\section{Results}

\section{Overall Patient Characteristics}

A total of 88 patients were available after a more than 1-year follow-up period (mean follow-up period: $5.7 \pm 3.8$ years; 5- and 10-year overall survival using the KaplanMeier method was 97.0 and $83.0 \%$, respectively). Of these, 12 patients under careful observation without medication and 1 patient who received only antifibrotic therapy (pirfenidone) were excluded (Fig. 1). The clinical characteristics of the remaining 75 patients with ARS-ILD who re- ceived anti-inflammatory therapy within 6 months after the ILD diagnosis are summarized in Table 1.

This anti-inflammatory therapy was continued during the follow-up period in all these patients. The improved group comprised 48 patients $(64.0 \%)$, the stable group 21 patients $(28.0 \%)$, and the worsened group 6 patients (8.0\%). During the follow-up period, $40.0 \%$ of the diagnoses included connective tissue diseases (CTDs) such as PM (10.7\%), DM (22.7\%), primary Sjögren syndrome (5.3\%), and systemic sclerosis-PM overlap (1.3\%). During their follow-up period (range: $0.75-9.0$ years), $11 \mathrm{pa}$ tients developed manifestations of CTD, and these patients were also included as CTD subjects. Forty-five other patients fulfilled the criteria for IPAF, including 30 patients with positive clinical domains, i.e., distal fissuring (13 patients), distal digital tip ulceration (2 patients), inflammatory arthritis or polyarticular morning joint stiffness $>60 \mathrm{~min}$ ( 7 patients), palmar telangiectasia ( $5 \mathrm{pa}-$ tients), Raynaud's phenomenon (5 patients), unexplained digital edema (4 patients), and unexplained fixed rash on the digital extensor surfaces (4 patients).

FVC was significantly lower in the patients in the improved group versus the other groups $(p=0.004)$, and 


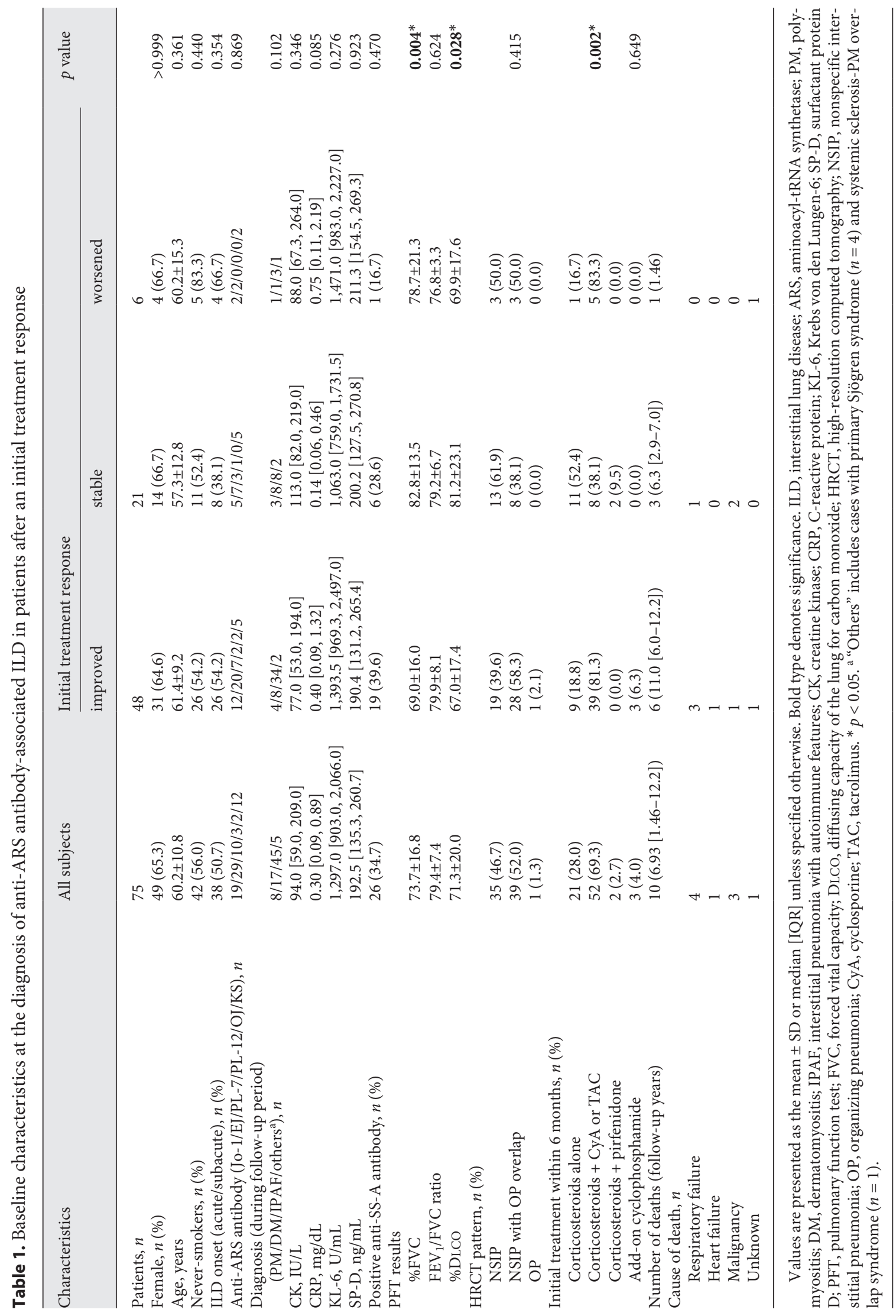


Table 2. Baseline characteristics at the diagnosis of anti-ARS antibody-associated ILD in the patients over the long-term course

\begin{tabular}{|c|c|c|c|c|}
\hline Patients, $n$ & 36 & 24 & 12 & \\
\hline Female, $n(\%)$ & $23(63.9)$ & $14(58.3)$ & $9(75.0)$ & 0.468 \\
\hline Age, years & $59.7 \pm 9.2$ & $58.9 \pm 9.6$ & $61.4 \pm 8.4$ & 0.442 \\
\hline Anti-ARS antibody (Jo-1/EJ/PL-7/PL-12/OJ/KS), $n$ & $9 / 14 / 6 / 2 / 0 / 5$ & $4 / 11 / 3 / 1 / 0 / 5$ & $5 / 3 / 3 / 1 / 0 / 0$ & 0.142 \\
\hline \multicolumn{5}{|l|}{ Diagnosis (during follow-up period) } \\
\hline$\left(\mathrm{PM} / \mathrm{DM} / \mathrm{IPAF} /\right.$ others $\left.^{\mathrm{a}}\right), n$ & $2 / 7 / 26 / 1$ & $1 / 3 / 19 / 1$ & $1 / 4 / 7 / 0$ & 0.336 \\
\hline $\mathrm{CK}, \mathrm{IU} / \mathrm{L}$ & $88.0[54.8,254.0]$ & $78.5[51.5,130.3]$ & $133.0[62.8,482.0]$ & 0.261 \\
\hline $\mathrm{CRP}, \mathrm{mg} / \mathrm{dL}$ & $0.30[0.09,1.21]$ & $0.19[0.09,0.76]$ & $0.48[0.09,1.61]$ & 0.650 \\
\hline $\mathrm{KL}-6, \mathrm{U} / \mathrm{mL}$ & $1,282.0[970.5,2,497.0]$ & $1,414.5[960.0,2,765.5]$ & $1,102.0[971.3,2,180.5]$ & 0.638 \\
\hline $\mathrm{FEV}_{1} / \mathrm{FVC}$ ratio & $79.9 \pm 8.4$ & $77.5 \pm 8.7$ & $84.7 \pm 5.5$ & $0.013^{*}$ \\
\hline$\%$ DLCO & $67.7 \pm 18.1$ & $69.9 \pm 17.9$ & $61.0 \pm 18.2$ & 0.233 \\
\hline \multicolumn{5}{|l|}{ Initial treatment within 6 months, $n(\%)$} \\
\hline Corticosteroids alone & $7(19.4)$ & $6(25.0)$ & $1(8.3)$ & \multirow[b]{2}{*}{0.384} \\
\hline Corticosteroids + CyA or TAC & $29(80.6)$ & $18(75.0)$ & $11(91.7)$ & \\
\hline Add-on cyclophosphamide & $2(5.6)$ & $0(0.0)$ & $2(16.7)$ & 0.105 \\
\hline
\end{tabular}

Values are presented as the mean \pm SD or median [IQR] unless specified otherwise. Bold type denotes significance. ARS, aminoacyl-tRNA synthetase; ILD, interstitial lung disease; PM, polymyositis; DM, dermatomyositis; IPAF, interstitial pneumonia with autoimmune features; CK, creatine kinase; CRP, C-reactive protein; KL-6, Krebs von den Lungen-6; SP-D, surfactant protein D; PFT, pulmonary function test; FVC, forced vital capacity; DLCO, diffusing capacity of the lung for carbon monoxide; CyA, cyclosporine; TAC, tacrolimus. ${ }^{*} p<0.05$. " "Others" includes 1 case of primary Sjögren syndrome $(n=1)$.

\%DLCO was significantly lower in the patients in the improved and worsened groups versus the stable group $(p=$ $0.028)$. As initial treatment with anti-inflammatory therapy within 6 months of diagnosis, corticosteroid use alone was more highly prevalent among the patients in the stable group, whereas the use of combination therapy (corticosteroids plus cyclosporine or tacrolimus) was more highly prevalent among the patients in the improved and worsened groups $(p=0.002)$.

The HRCT pattern included NSIP with OP overlap in $52.0 \%$, NSIP in $46.7 \%$, and OP in $1.3 \%$ of the cases, and no patients had usual interstitial pneumonia. Interobserver agreement on the HRCT pattern was good $(\kappa=$ 0.886 ; 95\% CI: $0.762-1.000$ ). The frequencies of the various HRCT findings are summarized in the online supplementary Table (for all online suppl. material, see www. karger.com/doi/10.1159/000488358). Most patients had findings such as GGO, reticulation, traction bronchiectasis, lower-lobe volume loss, and lower-lobe predominance.
Patient Characteristics in the Improved Group after Initial Anti-Inflammatory Therapy according to Changes in Available PFT Results over the 3-Year Follow-Up Period

Of the 48 patients in the improved group with PFT results at 1 year, 12 patients were excluded because of insufficient follow-up data, such as there being no available PFT results over the 3-year follow-up period. The remaining 36 patients were classified into two subgroups according to the PFT changes at 3 years compared to the results obtained at 1 year: a stabilization group $(n=24)$ and an unstable group $(n=12)$. As shown in Table 2 , the serum surfactant protein $\mathrm{D}$ level was significantly lower in the unstable group $(p=0.004)$, and the $\mathrm{FEV}_{1} / \mathrm{FVC}$ ratio was significantly lower in the stabilization group $(p=$ $0.013)$. HRCT findings of traction bronchiectasis in the right middle lobe or lingual lobe (known as middle lobe traction bronchiectasis) were more frequent in the unstable group $(p=0.033)$ (Table 3$)$. 
Table 3. HRCT findings of anti-ARS antibody-associated interstitial lung disease in the patients over the long-term course

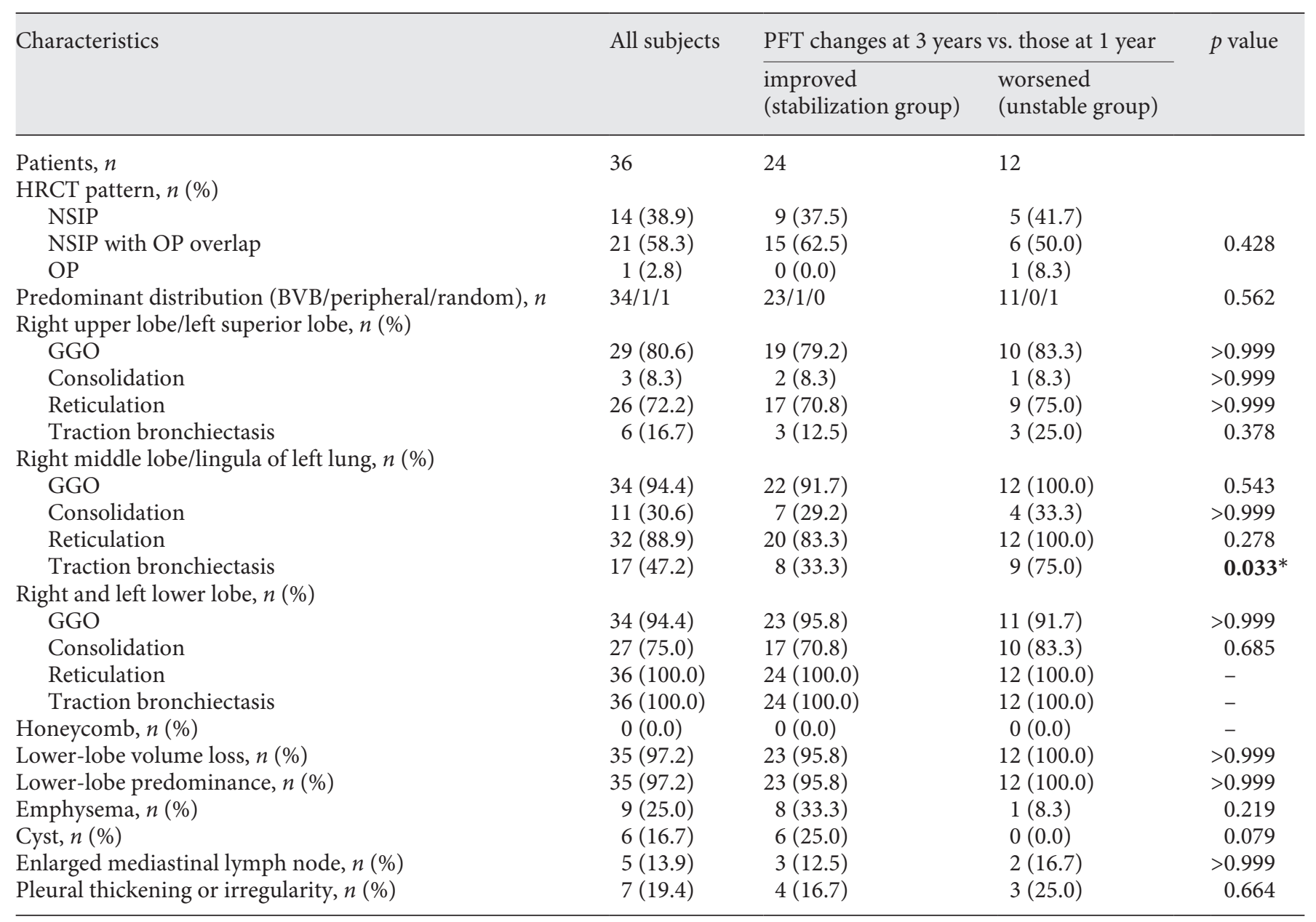

Bold type denotes significance. ARS, aminoacyl-tRNA synthetase; PFT, pulmonary function test; HRCT, high-resolution computed tomography; NSIP, nonspecific interstitial pneumonia; OP, organizing pneumonia; BVB, bronchovascular bundle; GGO, ground-glass opacity. ${ }^{*} p<0.05$.

Factors Predictive of Response to Initial

Anti-Inflammatory Therapy according to

\section{1-Year PFT Results}

Lower FVC or \%DLCO and combination therapy (corticosteroids plus cyclosporine or tacrolimus) were significant predictors of improvement over the initial course by univariate logistic analysis. However, a negative finding of lower-lobe volume loss was a significant predictor of worsening over the initial course. None of these factors remained a significant predictor in the multivariate logistic regression analysis (Table 4).
Factors Predictive of Long-Term Deterioration (Unstable Group) in the Improved Group after Initial Anti-Inflammatory Therapy

By univariate logistic analysis, a smoking habit, lower level of surfactant protein $\mathrm{D}$, higher $\mathrm{FEV}_{1} / \mathrm{FVC}$ ratio, and middle lobe traction bronchiectasis were significant predictors of unstable disease over the long-term clinical course (Table 5). Of these factors, middle lobe traction bronchiectasis was the only significant factor predictive of unstable disease by multivariate logistic analysis $(p=$ 0.041 ). As shown in Figure 2, specific patients with ARSILD with middle lobe traction bronchiectasis showed improvement with initial therapy, but afterwards they deteriorated as radiologically identified fibrotic disease ex- 
Table 4. Multivariate analysis of factors predictive of the initial response (improved and worsened) $(p<0.1$ in univariate analysis)

\begin{tabular}{|c|c|c|c|}
\hline \multirow[t]{2}{*}{ Variable } & \multicolumn{3}{|l|}{ Improved } \\
\hline & Odds ratio & $95 \% \mathrm{CI}$ & $p$ value \\
\hline \multicolumn{4}{|l|}{ PFT results } \\
\hline$\% \mathrm{FVC}$ & 0.970 & $0.926-1.016$ & 0.203 \\
\hline$\%$ DLCO & 0.995 & $0.955-1.037$ & 0.825 \\
\hline \multicolumn{4}{|l|}{ HRCT findings } \\
\hline \multicolumn{4}{|l|}{ Right upper lobe/left superior lobe } \\
\hline GGO & 1.990 & $0.532-7.439$ & 0.307 \\
\hline Reticulation & 1.553 & $0.432-5.575$ & 0.500 \\
\hline \multicolumn{4}{|l|}{ Right and left lower lobe } \\
\hline Consolidation & 1.901 & $0.531-6.805$ & 0.324 \\
\hline Lower-lobe volume loss & 6.803 & $0.574-80.560$ & 0.128 \\
\hline \multicolumn{4}{|l|}{ Initial treatment within 6 months } \\
\hline Corticosteroids alone & 1.000 & reference & \\
\hline Corticosteroids + CyA or TAC & 2.449 & $0.616-9.735$ & 0.203 \\
\hline \multirow[t]{2}{*}{ Variable } & \multicolumn{3}{|l|}{ Worsened } \\
\hline & Odds ratio & $95 \% \mathrm{CI}$ & $p$ value \\
\hline \multicolumn{4}{|l|}{ HRCT findings } \\
\hline Lower-lobe volume loss & 0.121 & $0.009-1.638$ & 0.112 \\
\hline Lower-lobe predominance & 0.500 & $0.013-19.562$ & 0.711 \\
\hline
\end{tabular}

CI, confidence interval; PFT, pulmonary function test; FVC, forced vital capacity; DLCO, diffusing capacity of the lung for carbon monoxide; HRCT, high-resolution computed tomography; GGO, ground-glass opacity; CyA, cyclosporine; TAC, tacrolimus.

tended over the long-term course. However, some patients without middle lobe traction bronchiectasis improved and remained stable over the long-term course (Fig. 3).

\section{Discussion}

The present study revealed that two-thirds of the patients with ARS-ILD who had received anti-inflammatory therapy showed improvement in their PFT results at 1 year, but one-third of these patients had deteriorated by 3 years. In other words, even in ARS-ILD patients showing a good response to initial therapy, pulmonary function frequently deteriorated during long-term follow-up. In terms of survival, only 2 patients died within 5 years from the ARS-ILD diagnosis and the 5-year survival rate was $97.0 \%$, which indicates relatively good survival of ARS-ILD patients, similar to that reported in previous studies $[6,7]$. Importantly, some patients with ARS-ILD suffered from refractory ILD over the long run, which led to a number of adverse events such as the necessity of home oxygen therapy and side effects related to the inability to reduce the dose of corticosteroids (e.g., muscle weakness, opportunistic infection, and diabetes mellitus).

Which subgroup of ARS-ILD patients showed longterm deterioration? The radiological analysis made in our study showed that middle lobe traction bronchiectasis was a significant predictor of long-term deterioration. Typical radiological findings for ARS-ILD are GGO, reticulation, traction bronchiectasis, lower-lobe volume loss, and disease predominantly distributed in the lower lobe $[9,18]$. The HRCT features were consistent with NSIP or NSIP with OP overlap in most of our subjects, though NSIP with OP overlap is not usually considered as a distinct HRCT pattern in ILD [19]. Although NSIP with OP overlap in rapidly progressive ARS-ILD may become resistant to therapy $[15,18]$, we could not definitively conclude this based on our relatively small cohort. Kotani et al. [20] reported that middle lobe GGO exten- 
Table 5. Factors predictive of long-term deterioration (unstable group) according to logistic regression analysis

\begin{tabular}{|c|c|c|c|c|c|c|}
\hline Variable & Odds ratio & $95 \% \mathrm{CI}$ & $p$ value & Odds ratio & $95 \% \mathrm{CI}$ & $p$ value \\
\hline Male sex & 0.467 & $0.100-2.173$ & 0.331 & & & \\
\hline Current or ex-smoker & 0.200 & $0.043-0.939$ & $0.041^{*}$ & 0.747 & $0.040-14.020$ & 0.845 \\
\hline Chronic form (ILD onset) & 0.500 & $0.118-2.116$ & 0.346 & & & \\
\hline PL-7 & 0.800 & $0.101-6.347$ & 0.833 & & & \\
\hline PL-12 & 0.800 & $0.037-17.196$ & 0.887 & & & \\
\hline Diagnosis (reference: PM) & 1.000 & reference & & & & \\
\hline $\mathrm{DM}$ & 1.333 & $0.057-31.121$ & 0.858 & & & \\
\hline IPAF & 0.368 & $0.020-6.723$ & 0.500 & & & \\
\hline$\%$ DLCO & 0.971 & $0.924-1.019$ & 0.229 & & & \\
\hline \multicolumn{7}{|l|}{ Initial treatment within 6 months } \\
\hline Corticosteroids alone & 1.000 & reference & & & & \\
\hline Corticosteroids + CyA or TAC & 3.667 & $0.388-34.368$ & 0.257 & & & \\
\hline HRCT pattern (reference: NSIP) & 1.000 & reference & & & & \\
\hline NSIP with OP overlap & 0.720 & $0.170-3.058$ & 0.656 & & & \\
\hline \multicolumn{7}{|l|}{ Right middle lobe/lingula of left lung } \\
\hline Traction bronchiectasis & 6.000 & $1.263-28.498$ & $0.024^{*}$ & 19.635 & $1.129-341.540$ & $0.041 *$ \\
\hline Emphysema & 0.182 & $0.020-1.667$ & 0.132 & & & \\
\hline
\end{tabular}

Bold type denotes significance. CI, confidence interval; ILD, interstitial lung disease; ARS, aminoacyl-tRNA synthetase; PM, polymyositis; DM, dermatomyositis; IPAF, interstitial pneumonia with autoimmune features; SP-D, surfactant protein D; FVC, forced vital capacity; DLCO, diffusing capacity of the lung for carbon monoxide; CyA, cyclosporine; TAC, tacrolimus; HRCT, high-resolution computed tomography; NSIP, nonspecific interstitial pneumonia; OP, organizing pneumonia. ${ }^{*} p<0.05$.

sion indicated a significantly poor prognosis for patients with acute or subacute ILD with DM. Following this report, because the disease distribution of ARS-ILD showed lower-lobe predominance, we hypothesized that detailed analysis not only of the lower lobe but also of the upper and middle lobes would be important. Notably, our study showed that middle lobe traction bronchiectasis was a predictor of poor long-term pulmonary function change, as shown in the examples in Figure 2. Debray et al. [9] mentioned that consolidations decrease or disappear in most cases but the disease may progress to fibrosis in more than one-third of patients. The presence of this finding with a varying extent of disease (i.e., consolidation, GGO, and reticulation) may be related to the possibility of irreversible pulmonary fibrosis. Interestingly, all 6 patients with worsened PFT results at 1 year had this finding (see the online suppl. Table). Because this finding led to a statistical error in the logistic regression analysis, it could not be determined whether this was a factor predictive of worsening over the initial course. Taken together, middle lobe traction bronchiectasis in ARS-ILD may indicate the possibility of a deterioration of pulmonary function in both the short and the long term. Conversely, even in instances of a wide extent of disease, a negative finding of middle lobe traction bronchiectasis in ARSILD may lead to a good response and long-term stabilization.

Our study could not discover a link between individual anti-ARS antibodies and the long-term clinical course of ILD, in contrast to previous reports on an association between worse prognosis and anti-PL-7 antibody [14, 21 . This is because the lower frequency of PM/DM and the higher frequency of IPAF in our cohort may have led to results different from those of previous studies. 
Many immunosuppressive drugs have been evaluated in ARS-ILD [22-24]. Previous studies have shown that combination therapy improved the findings of PFTs and chest HRCT scans in acute or subacute progressive PM/ DM-ILD [22, 25-27]. We previously reported the importance of combination therapy, which should be on the list of options to prevent relapse in anti-EJ ILD [10]. However, it is controversial whether all ARS-ILD patients need corticosteroids in addition to the other immunosuppressive drugs they are taking. In our study, this combination therapy was a significant predictor of initial improvement in the univariate analysis, but it had no influence on long-term change in pulmonary function. Although careful management is required due to the potential risk of opportunistic infection, our findings suggest that a more aggressive therapy may be required for
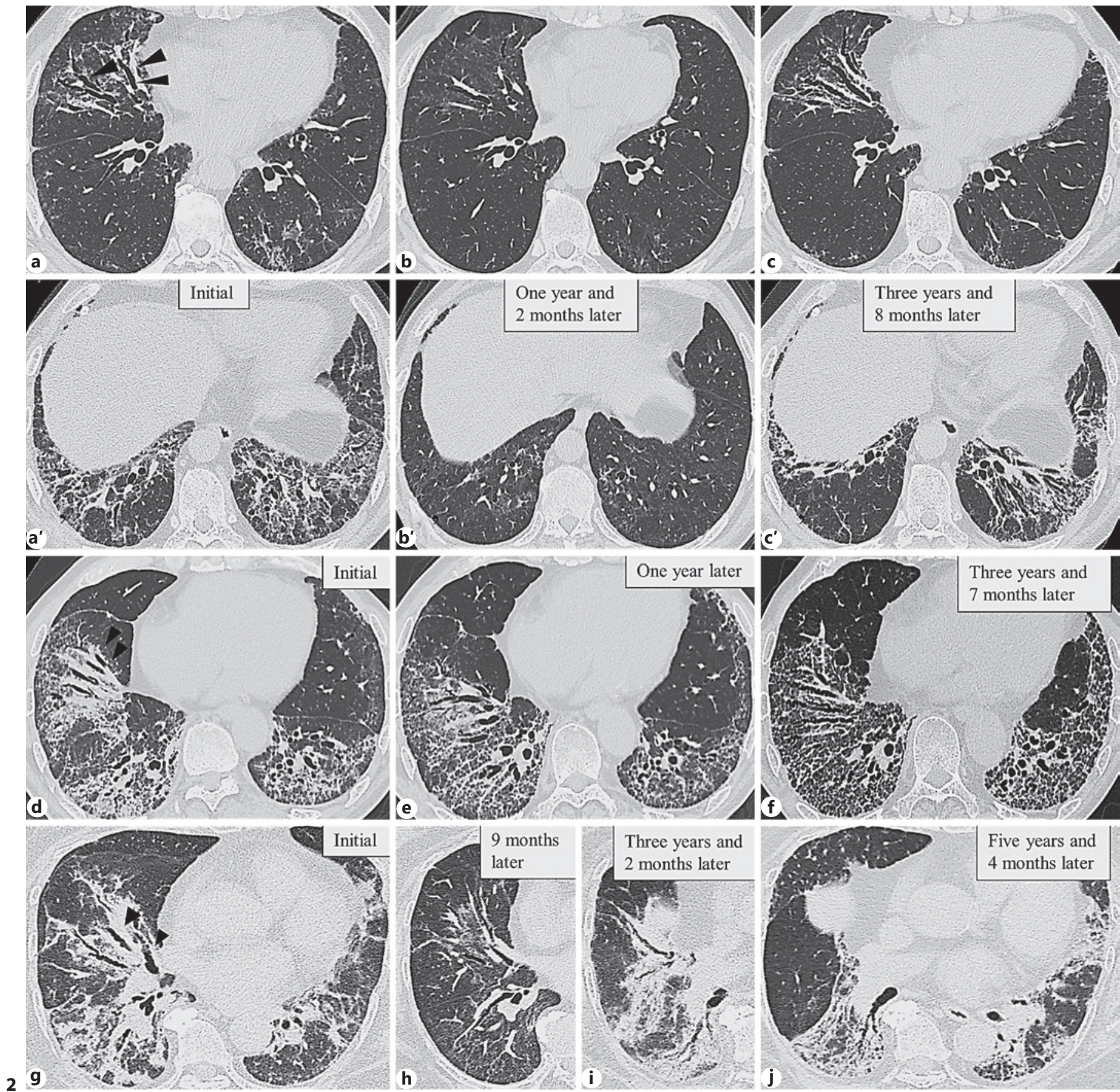

(For legend see next page.) 
ARS-ILD patients presenting with middle lobe traction bronchiectasis because of the possibility of a poor outcome of ILD over the long run.

Our study has two limitations. First, it is a single-institution, retrospective study. Second, selection bias may be present, because this study was performed in a single center specializing in respiratory disease and some of the pa- tients were excluded from the long-term analysis because of insufficient follow-up data.

In conclusion, most patients with ARS-ILD receiving anti-inflammatory therapy improved or remained stable in the first year. However, over the long run, the course of some patients deteriorated despite showing improvement initially. Even though the extent of disease is limit-
Fig. 3. Patients without traction bronchiectasis in the middle or lingual lobe. a Highresolution computed tomography (HRCT) scan of a 40-year-old woman positive for anti-EJ antibody showing ground-glass opacities (GGOs) with reticulation and traction bronchiectasis in the lower lobes, but these lesions were not seen in the middle lobe and lingula. b The same patient 3 years and 4 months later. HRCT showed improvement of the GGOs and disease extension. c HRCT scan of a 50-year-old woman positive for anti-PL-7 antibody showing GGOs predominantly in the lower lung. d The same patient 2 years and 5 months later. HRCT showed marked improvement of the abnormal shadows. e HRCT scan of a 56-year-old man positive for anti-Jo-1 antibody showing consolidation along with bronchovascular bundle and lower-lobe volume loss, findings compatible with nonspecific interstitial pneumonia with an organizing pneumonia overlap pattern. $f$ The same patient 2 years and 6 months later. HRCT showed dramatic improvement of the abnormal findings.

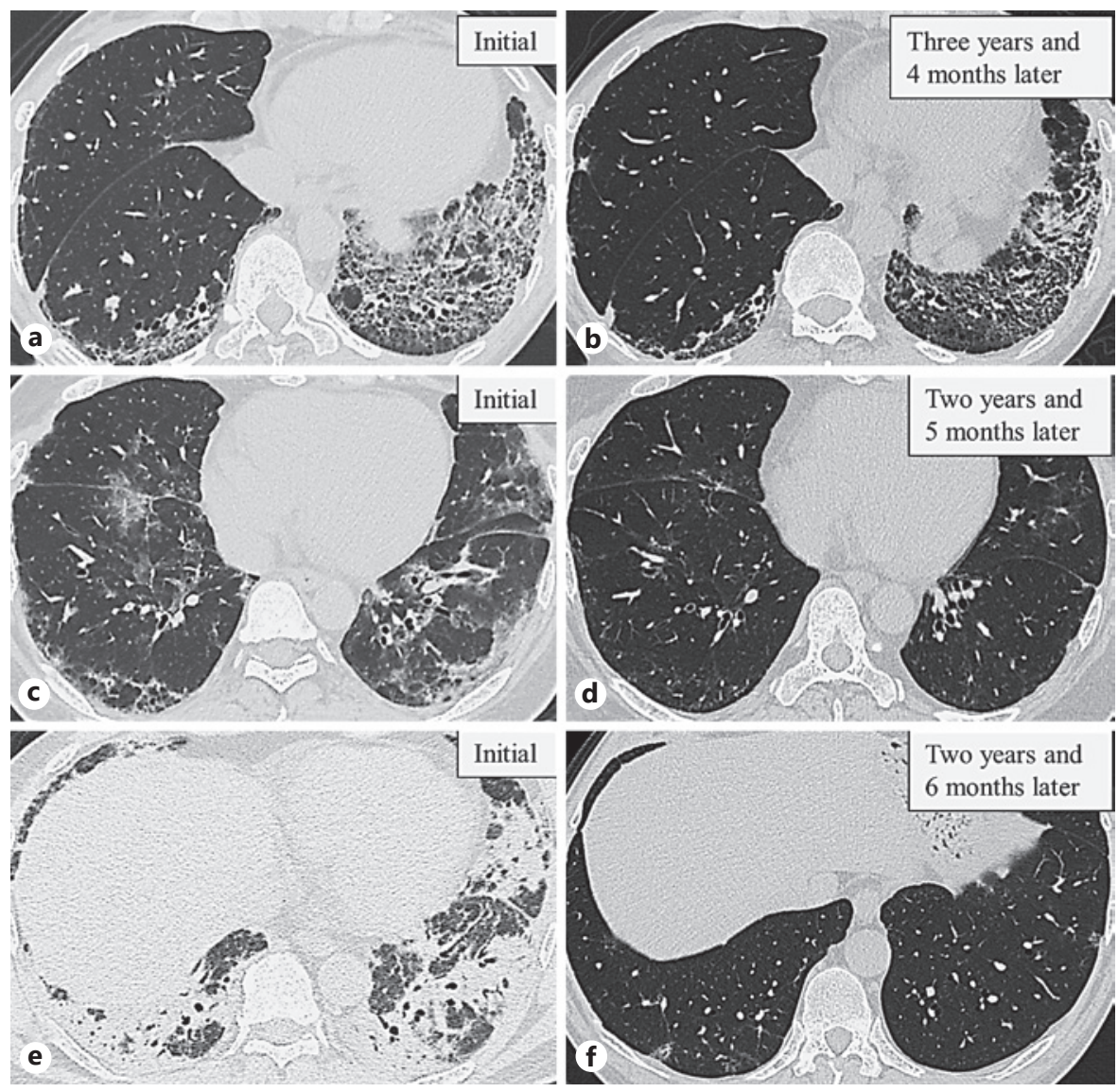

Fig. 2. Middle lobe traction bronchiectasis. a-c Radiological timedependent changes in a 58-year-old man with anti-Jo-1 antibody and a nonspecific interstitial pneumonia (NSIP) pattern. a, a' At the initial diagnosis, high-resolution computed tomography (HRCT) showed ground-glass opacities (GGOs) with subpleural sparing predominantly in the lower lung, and traction bronchiectasis in the middle lobe (arrowheads) and lower lobe. This traction bronchiectasis represents irregular bronchial dilatation caused by surrounding pulmonary fibrotic change. $\mathbf{b}, \mathbf{b}^{\prime}$ One year and 2 months later, HRCT showed a marked improvement of the traction bronchiectasis and GGOs. c, $\mathbf{c}^{\prime}$ Three years and 8 months after the initial diagnosis, HRCT showed exacerbation mainly with reticulation, GGOs, and traction bronchiectasis, which suggested fibrotic changes. d-f Radiological time-dependent changes in a 72-year-old man with anti-EJ antibody and NSIP with an organiz- ing pneumonia (OP) overlap pattern. d At the initial diagnosis, HRCT showed consolidations superimposed on GGOs with traction bronchiectasis (arrowheads: middle lobe traction bronchiectasis). e One year later, the consolidations had resolved, but GGOs with reticulation remained. $\mathbf{f}$ Three years and 7 months later, HRCT showed a moderate increase in disease extent of the reticulation and traction bronchiectasis. $\mathbf{g}-\mathbf{j}$ Radiological time-dependent changes in a 63-year-old woman with anti-PL-7 antibody and NSIP with an OP overlap pattern. g At the initial diagnosis, HRCT showed mainly consolidation with GGOs with traction bronchiectasis (arrowheads). $\mathbf{h}$ Nine months later, the consolidations had disappeared. i Three years and 2 months later, the consolidations had relapsed. j Five years and 4 months later, HRCT showed regions of consolidation with lower-lobe volume loss. 
ed, a positive radiological finding of middle lobe traction bronchiectasis in ARS-ILD could be a useful predictor of poor long-term disease behavior. This radiological finding may also be helpful in assessing appropriate strategies to treat ARS-ILD.

\section{Acknowledgments}

We sincerely thank Ms. Etsuko Iwata and Ms. Miyako Nakagawa of the Tokai University School of Medicine for their contribution to detecting the anti-ARS antibodies and Yosuke Sasaki of
Satista Co., Ltd., for his advice on statistical analysis. The authors would like to thank Rise Japan LLC for the professional English language review.

\section{Statement of Ethics}

This retrospective cohort study was approved by the institutional review board of the Kanagawa Cardiovascular and Respiratory Center (KCRC-17-0014). Because of the retrospective nature of the study, the review board waived the need for written informed consent from the patients.

\section{References}

-1 Mimori T, Imura Y, Nakashima R, Yoshifuji $\mathrm{H}$ : Autoantibodies in idiopathic inflammatory myopathy: an update on clinical and pathophysiological significance. Curr Opin Rheumatol 2007;19:523-529.

2 Hamaguchi Y, Fujimoto M, Matsushita T, Kaji K, Komura K, Hasegawa M, Kodera M, Muroi E, Fujikawa K, Seishima M, Yamada H, Yamada R, Sato S, Takehara K, Kuwana M: Common and distinct clinical features in adult patients with anti-aminoacyl-tRNA synthetase antibodies: heterogeneity within the syndrome. PLoS One 2013;8:e60442.

-3 Fischer A, Lee JS, Cottin V: Interstitial lung disease evaluation: detecting connective tissue disease. Respiration 2015;90:177-184.

-4 Takato H, Waseda Y, Watanabe S, Inuzuka K, Katayama N, Ichikawa Y, Yasui M, Fujimura M: Pulmonary manifestations of anti-ARS antibody positive interstitial pneumonia with or without PM/DM. Respir Med 2013; 107:128-133

5 Fischer A, Swigris JJ, du Bois RM, Lynch DA, Downey GP, Cosgrove GP, Frankel SK, Fernandez-Perez ER, Gillis JZ, Brown KK: Antisynthetase syndrome in ANA and anti-Jo-1 negative patients presenting with idiopathic interstitial pneumonia. Respir Med 2009;103 1719-1724.

-6 Zamora AC, Hoskote SS, Abascal-Bolado B, White D, Cox CW, Ryu JH, Moua T: Clinical features and outcomes of interstitial lung disease in anti-Jo-1 positive antisynthetase syndrome. Respir Med 2016;118:39-45.

-7 Mejía M, Herrera-Bringas D, Pérez-Román DI, Rivero H, Mateos-Toledo H, CastorenaGarcía P, Figueroa JE, Rojas-Serrano J: Interstitial lung disease and myositis-specific and associated autoantibodies: clinical manifestations, survival and the performance of the new ATS/ERS criteria for interstitial pneumonia with autoimmune features (IPAF) Respir Med 2017;123:79-86.
8 Tillie-Leblond I, Wislez M, Valeyre D, Crestani $B$, Rabbat $A$, Israel-Biet $D$, Humbert $M$, Couderc LJ, Wallaert B, Cadranel J: Interstitial lung disease and anti-Jo-1 antibodies: difference between acute and gradual onset. Thorax 2008;63:53-59.

-9 Debray MP, Borie R, Revel MP, Naccache JM, Khalil A, Toper C, Israel-Biet D, Estellat C, Brillet PY: Interstitial lung disease in antisynthetase syndrome: initial and follow-up CT findings. Eur J Radiol 2015;84:516-523.

10 Sasano H, Hagiwara E, Kitamura H, Enomoto Y, Matsuo N, Baba T, Iso S, Okudela K, Iwasawa T, Sato S, Suzuki Y, Takemura T, Ogura $\mathrm{T}$ : Long-term clinical course of anti-glycyl tRNA synthetase (anti-EJ) antibody-related interstitial lung disease pathologically proven by surgical lung biopsy. BMC Pulm Med 2016;16:168.

11 Bohan A, Peter JB: Polymyositis and dermatomyositis (first of two parts). N Engl J Med 1975;292:344-347.

12 Fischer A, Antoniou KM, Brown KK, Cadranel J, Corte TJ, du Bois RM, Lee JS, Leslie KO, Lynch DA, Matteson EL, Mosca M, Noth I, Richeldi L, Strek ME, Swigris JJ, Wells AU, West SG, Collard HR, Cottin V; ERS/ATS Task Force on Undifferentiated Forms of CTD-ILD: An official European Respiratory Society/American Thoracic Society research statement: interstitial pneumonia with autoimmune features. Eur Respir J 2015;46:976987.

13 Fujisawa T, Hozumi H, Kono M, Enomoto N, Hashimoto D, Nakamura Y, Inui N, Yokomura K, Koshimizu N, Toyoshima M, Shirai T, Yasuda K, Hayakawa H, Suda T: Prognostic factors for myositis-associated interstitial lung disease. PLoS One 2014;9:e98824.

- 14 Fujisawa T, Hozumi H, Kono M, Enomoto N, Nakamura Y, Inui N, Nakashima R, Imura Y, Mimori T, Suda T: Predictive factors for longterm outcome in polymyositis/dermatomyositis-associated interstitial lung diseases. Respir Investig 2017;55:130-137.
15 Travis WD, Costabel U, Hansell DM, King TE Jr, Lynch DA, Nicholson AG, et al; ATS/ERS Committee on Idiopathic Interstitial Pneumonias: An official American Thoracic Society/European Respiratory Society statement: update of the international multidisciplinary classification of the idiopathic interstitial pneumonias. Am J Respir Crit Care Med 2013;188:733-748.

16 Hansell DM, Bankier AA, MacMahon $\mathrm{H}$ McLoud TC, Müller NL, Remy J: Fleischner Society: glossary of terms for thoracic imaging. Radiology 2008;246:697-722.

17 Kondoh Y, Taniguchi H, Kitaichi M, Yokoi T, Johkoh T, Oishi T, Kimura T, Nishiyama O, Kato K, du Bois RM: Acute exacerbation of interstitial pneumonia following surgical lung biopsy. Respir Med 2006;100:1753-1759.

18 Waseda Y, Johkoh T, Egashira R, Sumikawa H, Saeki K, Watanabe S, Matsunuma R, Takato H, Ichikawa Y, Hamaguchi Y, Shiraki A, Muro Y, Yasui M, Prosch H, Herold C, Kasahara K: Antisynthetase syndrome: pulmonary computed tomography findings of adult patients with antibodies to aminoacyl-tRNA synthetases. Eur J Radiol 2016;85:1421-1426.

19 Nicholson AG, Colby TV, Wells AU: Histopathological approach to patterns of interstitial pneumonia in patient with connective tissue disorders. Sarcoidosis Vasc Diffuse Lung Dis 2002;19:10-17.

20 Kotani T, Takeuchi T, Yoshimatsu Y, Ishida T, Yamamoto N, Fujiki Y, Oda K, Isoda K, Hata K, Kamimori T, Fujiwara H, Makino S, Hanafusa T: Initial limited three-level thinsection computed tomography scorings predict the prognosis of acute/subacute interstitial pneumonia in patients with dermatomyositis. Mod Rheumatol 2016;26:738-743.

21 Shi J, Li S, Yang H, Zhang Y, Peng Q, Lu X, Wang G: Clinical profiles and prognosis of patients with distinct antisynthetase autoantibodies. J Rheumatol 2017;44:1051-1057. 
-22 Wilkes MR, Sereika SM, Fertig N, Lucas MR, Oddis CV: Treatment of antisynthetase-associated interstitial lung disease with tacrolimus. Arthritis Rheum 2005;52:2439-2446.

23 Marie I, Hatron PY, Dominique S, Cherin P, Mouthon L, Menard JF: Short-term and longterm outcomes of interstitial lung disease in polymyositis and dermatomyositis: a series of 107 patients. Arthritis Rheum 2011;63:34393447.

24 Andersson H, Sem M, Lund MB, Aaløkken TM, Günther A, Walle-Hansen R, Garen T,
Molberg Ø: Long-term experience with rituximab in anti-synthetase syndrome-related interstitial lung disease. Rheumatology (Oxford) 2015;54:1420-1428.

25 Maeda K, Kimura R, Komuta K, Igarashi T: Cyclosporine treatment for polymyositis/dermatomyositis: is it possible to rescue the deteriorating cases with interstitial pneumonitis? Scand J Rheumatol 1997;26:24-29.

26 Kameda H, Nagasawa H, Ogawa H, Sekiguchi $\mathrm{N}$, Takei H, Tokuhira M, Amano K, Takeuchi $\mathrm{T}$ : Combination therapy with corticosteroids, cyclosporin A, and intravenous pulse cyclophosphamide for acute/subacute interstitial pneumonia in patients with dermatomyositis. J Rheumatol 2005;32:1719-1726.

27 Kotani T, Takeuchi T, Makino S, Hata K, Yoshida S, Nagai K, Wakura D, Shoda T, Hanafusa T: Combination with corticosteroids and cyclosporin-A improves pulmonary function test results and chest HRCT findings in dermatomyositis patients with acute/subacute interstitial pneumonia. Clin Rheumatol 2011; 30:1021-1028. 